\title{
Contributing to the Modeling of a Multi-Agent System for Comfort Control in Smart Buildings Applications
}

\author{
Ghezlane Halhoul Merabet \\ ENSIAS, Mohammed V University \\ of Rabat, Morocco
}

\author{
Mohamed Essaaidi \\ ENSIAS, Mohammed V University \\ of Rabat, Morocco
}

\author{
Driss Benhaddou \\ College of Technology, University \\ of Houston, TX, USA
}

\begin{abstract}
The introduction of new technologies has been adopted as a strategy in the search for better energy efficiency, better environmental quality for users and reduction of environmental impacts. Such examples of this strategy are the installation of automation systems and/or their integration with existing systems in buildings. Regarding the environmental quality, studies related to the thermal comfort show that temperatures outside a comfort zone can affect the productivity and well-being of the users. Knowing that, in most cases, the temperature control of the environment is performed through types of equipment that have a significant share in the energy consumption of buildings, the motivation to develop researches that contribute to proposals and discussions of solutions for better energy efficiency while maintaining the thermal comfort of users. In this regard, this paper describes the use of ambient intelligence as a solution to model and control the behavior of a system involved in building automation and contributing to a significant optimization in terms of comfort and energy saving.
\end{abstract}

\section{General Terms}

Multi-Agent Systems, Thermal Comfort

\section{Keywords}

Smart buildings, Multi-Agent Systems, Thermal comfort, JADE platform

\section{INTRODUCTION}

An intelligent building (or environment) can be defined as the one that uses computer technology to automatically control its operation, in such a way as to optimize the users' comfort, the resources consumption (for example energy), safety and work efficiency" [1].

In fact, the increasing interest in building automation is not a coincidence. Today, there is a wide variety of low-cost devices allowing the interconnection of electrical devices, sensors and computers within a building. The idea thus emerged to make more appropriate use of these resources, in such a way to reduce the costs of operation and maintenance of the building and improving the comfort and safety of its occupants. However, the rational use of these resources often requires that the computer software involved have a degree of flexibility and autonomy that can hardly be achieved through classical design and programming techniques. In this case, it refers to systems that should be intelligent enough to act appropriately in changing, unpredictable and complex situations.

The problems with these characteristics have usually been addressed through the intelligent systems, including both Soft Computing systems and classical approaches of artificial intelligence based on representations and methods of highlevel reasoning [2]. Besides the significant differences between these approaches, there is a unifying concept known as intelligent agents that has allowed the use of the most appropriate technique in each case and perform various forms of hybridization in cases where necessary [3], [4].

In general, an intelligent agent is an autonomous entity (software or hardware), directly connected to the problem environment, which is capable of exhibiting flexible behavior (reactive, proactive and social) tending to meet its design objectives. If there is more than one agent interacting in a shared environment, the resulting multi-agent system has considerable added value since agents can share their knowledge and skills, being able to perform activities that go beyond their individual capabilities.

The problematic associated to the smart buildings' management, has many characteristics that are appropriate to be approached with a multi-agent approach, as it has been reflected in several works related to the analysis, development, and implementation of systems for the autonomous and intelligent management of buildings for residential use, offices and industrial ones. From this tendency, a considerable number of theoretical and practical challenges have emerged to which the paradigm of multiagent systems should respond according to the particularities of this application area. Thus, aspects such as planning, optimization of the use of resources, scheduling, machine learning, communication and coordination of agents begin to be reconsidered from the perspective of intelligent management of a building.

In this context, this paper describes in section 2 some general aspects of intelligent buildings. Section 3 introduces elementary concepts of multi-agent systems and their applicability in smart buildings. Section 4 presents the related works, then section 5 portrays our contribution. Finally, section 6 presents conclusions.

\section{SMART BUILDING: A MULTIDISCIPLINARY ENTITY}

In the last century, the percentage of the world's population living in the cities has jumped from $10 \%$ to more than $50 \%$, and it is expected that by $2050,75 \%$ of this population will be living in cities. Throughout the early 21 st century, big cities have brought to the fore the enormous challenge of sustainable development, which has been defined as the need to guarantee the current requirements, without compromising the ability of future generations to meet their own needs [5]. As a consequence of the need to guarantee comfort and resources to a world urban population, buildings are today the largest energy consumers in the world, accounting for $40 \%$ of world energy consumption and consuming $50 \%$ of the final electricity available in the world [6].

The urban environment is the main platform for the introduction of technologies and policies that can contribute to 
reducing energy consumption and increasing the energy efficiency of available services. However, buildings are the main consumer of energy in cities, being the focus of several researches and applications developed to ensure the comfort of users in a more efficient way.

As was pointed out in [7], the "Smart Buildings" (SB) term has been used for more than two decades. During the second half of the 1970s, the term referred to buildings that were built based on the concepts of energy efficiency; in 1980, it started to be used to refer to buildings that could be controlled through a computer. In this regard, technological advances have made it possible to build buildings capable of managing their infrastructure and services - such as lighting or air conditioning systems - in a more efficient and autonomous way, using some pre-defined configurations via software as a basis for decision making.

Besides, a new concept of applications arises due to advances in the fields of electronics and communication systems. Over the last decade, the development of devices and equipment has sought new forms of interconnection between the physical and digital worlds.

Indeed, the combination of computational power and communication networks has made it possible to develop applications capable of perceiving and interpreting the real world. Through sensors, analog signals present in the real world are converted into digital data in order to be processed locally or transmitted to any computational source of processing. The analysis of these data allows taking actions in the real world by means of actuators, characterizing a feedback control able to manage the dynamics of several systems. The advancement of Information and Computer Technology (ICT) and the emergence of concepts such as the Internet of Things (IoT) enabled to add intelligence to digital systems solutions.

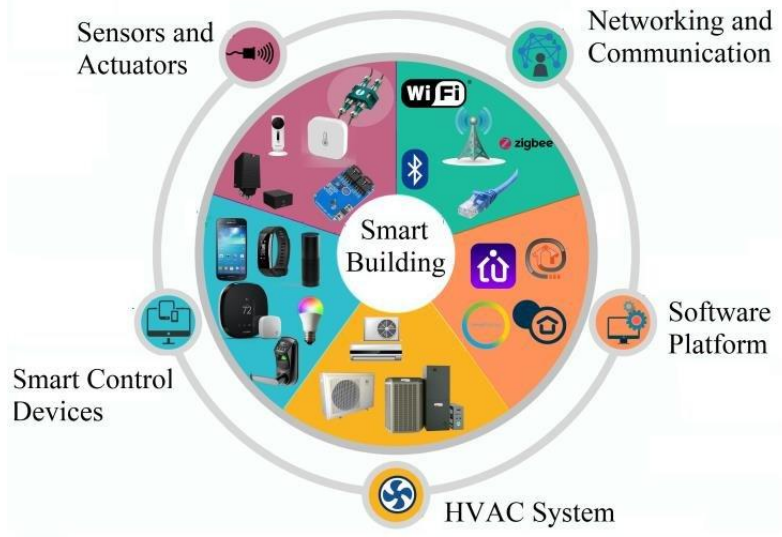

Fig 1: Smart Building Components [8].

As described in [9], the definition of a smart building encompasses the set of dynamic and intelligent systems installed in a building, capable of storing, producing, sharing, analyzing and acting based on the collected data. The installed system is capable of increasing the energy efficiency of its actions over time through learning from the actions that produced the best results. In addition, the system is capable of optimizing performance in different aspects: safety, energy efficiency, productivity, efficient operation, efficient consumption, among others.

In addition to the benefits delivered to direct users, smart buildings will intensify the existing relationships between buildings and the city. The bilateral exchange of energy between building and city will be a consequence of the technological advancement of smart electricity grids, known as Smart Grid. As analyzed by [10], smart grids integrate information and communication technologies with the current power grid, enabling new ways of communication between users and distribution utilities.

In addition, with the emergence and use of smart electricity meters, new sources of electricity will be introduced through distributed generation. Once connected to the city's infrastructure, buildings take on the role of consumergenerator, contributing to increased power quality and accelerating the response time of the electricity system at peak times. Based on the positive aspects of each of the definitions found in the literature, in this context, we may define a smart building as a building that provides comfort, safety and the necessary resources to users through dynamic systems that are responsive, intercommunicable with each other and integrated into the city. Its systems interact with users, anticipating their needs and providing information about the use of their utilities.

\section{MULTI-AGENT SYSTEMS AND SMART BUILDINGS}

An intelligent agent is an autonomous entity (software or hardware), directly connected to the problem environment, which is capable of exhibiting flexible behavior tending to meet its design objectives. The flexible behavior refers to the fact that it should be able to react in time to changes in the environment (reactivity), take the initiative when necessary (pro-activity) and communicate and interact with other human or artificial agents (sociability). If several agents interact in a shared environment, it is then a multi-agent system [11]

A multi-agent system (MAS) is a tightly coupled network of components (agents) working together to solve problems beyond their individual capabilities and/or knowledge. Some of the features characterizing MAS are:

- Agents are autonomous, distributed, possibly heterogeneous and can be individual or cooperative;

- Each agent has incomplete information, or limited capabilities to solve the problem, so each agent has a limited point of view;

- A MAS is usually open and there is no centralized design;

- There is no global control of the system and the data is decentralized;

- Asynchronous computation;

- There is some infrastructure that specifies communication and interaction protocols.

There are several reasons that enhance the growing use of Multi-Agent Systems, such as:

- Robustness;

- Efficiency;

- Ability to allow the interoperation of existing legacy systems;

- Ability to solve problems in which the data or control skills are distributed;

- Ability to solve problems that require sophisticated patterns of interaction between system components. 
In this regard, multi-agent systems are the most available approach to undertaking complex systems. Their decentralized nature makes them particularly suitable for such systems. Then Multi-Agent Systems allow working on the overall operation of a system by focusing on the entities comprising it and their interactions. Hence, it is actually possible to visualize more clearly the relationships existing between the intelligent building management and MAS. In this case, we can have agents that represent different devices, sensors or actuators, building's occupants, rooms, and even the entire building. It will also be possible to design the agents considering a functional vision and have agents of personal comfort, security, individualization, and site of people, etc.

Agents may be distributed on different computers and devices, collecting the information required to perform their tasks and communicating when necessary. Each agent shall be as autonomous as possible, requiring the minimum human intervention for its operation. Some agents will perform continuously, such as those associated with rooms or other parts of the building. Others, on the other hand, will have a shorter life cycle according to needs. This will be the case of the agents representing the people occupying the building, which may be created/destroyed each time the associated person enters/leaves the building.

The characteristics and properties of multi-agent systems can play a fundamental role in achieving those attributes that we previously considered desirable for a system that controls an intelligent building (flexibility, scalability, robustness, conviviality and reactivity).

An intelligent agent must, by definition, be able to exhibit a flexible behavior. The flexibility that an agent can provide in this context is not limited to the facilities for defining new policies and devices. In this case, the meaning is broader and may include behaviors and interactions of agents with a significant degree of complexity. The scalability is guaranteed, since the incorporation of new components only involves the definition and creation of the agents representing them. The robustness is implicit in the distributed characteristics of the MAS. If a computer fails, the affected agents may be replaced by other agents who adopt their roles, thus reducing the problems associated with the critical failure points. On the other hand, an agent will be able to anticipate the needs of a user, take the initiative and execute a task autonomously without the active requirement of a person. In the ideal case of autonomy, this agent will be able to learn the person's particular preferences and perform his actions according to his observations of his behavior. Also, it will be able to respond urgently when the situation requires using a suitable agent architecture for these cases. These characteristics can positively influence all aspects of conviviality.

Regarding the communication between the agents controlling an intelligent building is also a fundamental factor to consider. In some cases, it has been based on traditional Distributed Artificial Intelligence approaches such as blackboard systems. It is also possible to modify the environment as a reactive form of communication. The current trend is to use agent communication languages based on the "speech acts" theory, such as $\mathrm{KQML}^{1}$ and $\mathrm{FIPA}^{2} \mathrm{ACL}^{3}$.

The coordination of agents, as in other domains, is an important aspect to achieve a consistent behavior of the MAS that manages an intelligent building. The distribution of data and control among several autonomous agents often leads to conflicts due to the existence of different points of view, access to scarce resources or incompatibility of objectives. Another crucial aspect to consider is the way in which the agents will represent and reason about the functioning of the devices, rooms, the building, the capacities of the other agents and the building occupants. In some cases, reactive schemes will be adequate to respond quickly to urgent demands. In others (e.g., if there is interaction with the building through natural language) deliberative agents with high level representation and reasoning capacities will be necessary.

\section{RELATED WORKS}

\subsection{Smart Building Optimization}

Works on intelligent buildings and home automation reporting the use of the multi-agent systems for intelligent energy/ comfort management has been appearing in the literature for many years. For example, in [12], they presented an energy management system, its control and simulation using MAS. The purpose of this work is to improve the management of energy and comfort in a commercial building. In the residential sector, new systems have been proposed to meet various challenges, such as implementing intelligent residential monitoring [13], providing customers with realtime feedback for better managing their consumption [14] and developing home automation [15]. For example, Abras et al. [16] proposed a multi-agent home automation system that controls appliances and energy sources in buildings.

Moreover, in Klein et al. [17] presented an agent-based system for comfort and energy management (MACES: MultiAgent Comfort and Energy System) by coordinating the building appliances and the occupants and using a multiobjective optimization MDP (Markov Decision Problems). Also, in [18]-[20] they adopted hierarchical MAS architecture to deal with comfort and energy efficiency management. The proposed agent-controllers are optimized by integrating PSO (Particle Swarm optimization) and MOGA (Multi-Objective Genetic Algorithm).

In addition, in [11] and [21] the authors proposed a technique based on a MAS for the control and real-time learning of elements of a smart home. Minar et al. [21] addressed, through their work, a wider vision on the construction of a distributed architecture for ubiquitous computing agents. A similar architecture is proposed in [11], although this latter approach focuses on the implementation details of the agents that mainly meet the needs of compactness since, in the first approach, these concepts are not taken into account. The MAS based on the oriented approach interactions were developed in [22] by coordinating a human machine interface (HMI) and agent-based communities, to optimize the comfort management as well as the heating, ventilation and air conditioning (HVAC) processes. 


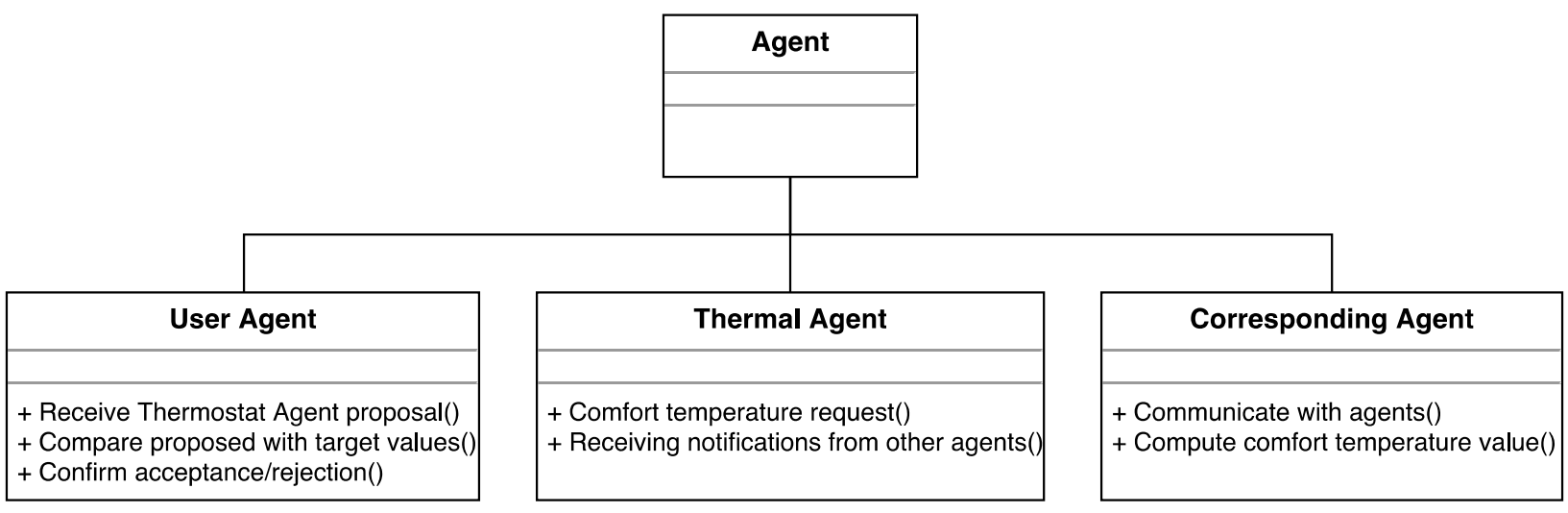

Fig 2: Class diagram of the Intelligent Monitoring System

\subsection{Human Behavior/Occupancy}

In residential context, various works have been performed on modeling and estimating buildings occupancy. In the context of the American Society of Heating, Refrigerating and Air Conditioning Engineers (ASHRAE), Claridge et al. [23] of the American Society of Heating, Refrigerating and Air Conditioning Engineers (ASHRAE), Claridge et al. [23] proposed using daily profiles to simulate different categories of buildings for professional use. With this objective, Liao and Barooah [24] developed an agent-based model to simulate occupant behavior in an office building. The proposed model has been validated with sensor data for a particular case of a room and an occupant. Based on statistical data over eight years of measurements, Haldi [25] developed an agent-based predictive model of the actions of office buildings occupants on windows. Variables include indoor and outdoor temperatures, the presence of rain, and occupant absences/presences. The model reflected individual characteristics such as air quality, thermal and visual comfort.

\subsection{Occupants' Modeling}

the user activity to optimize the energy performance. Such example in [26], in which a probabilistic model proposed, based on Markov processes representing time schedules in residential building, proposed an easy-to-access MAS for nonspecialist experts. However, it takes into account only one dimension (the activity), ignoring the thermal issues of the building, the individual comfort, the collective organization of the home, etc. In contrast, other studies focus on thermal comfort [27], occupant actions on the building [28].

In this regard, the work presented by Kashif [29] on the design of a detailed human behavior model for energy management. Based on the analysis of daily activities, the author proposed a behavioral model of the inhabitants based on BDI (Belief Desire Intention) agents that also takes into account the thermal properties of the building and the appliances consumption. In this model, the behavior of the inhabitants is represented through a reactive and deliberative decision-making process, based on Brahms architecture [30]. Such approach is interesting as it is based on real activity data obtained in real-life situations.

\section{CASE STUDY: AGENT-BASED COMFORT CONTROL IN SMART BUILDINGS}

The objective of studying the people behavior in certain environmental and personal conditions is to predict the degree of satisfaction/dissatisfaction with the thermal environment. In this context, we are interested in the thermal comfort within office buildings. Indeed, we have implemented an agent-based prototype acting as an expert system that assists in the management of the building to improve the quality of services for the occupants. The scenario took place in a lab at the College of Technology at the University of Houston. In order to be able to build a profile of temperature, thermal perception for certain people, we shall first discover the ideal comfort temperature standard for the user based on its preference and sensations.

\subsection{The proposed Multi-Agent System}

The proposed system allows improving the social environments where the users take part to improve their thermal comfort. An additional feature is to record user preferences for statistical purposes to improve the conditions of the environments. The prototype implemented in this work is composed of the following agents provided by JADE (Java Agent Development) framework [31]:

- $\quad$ AMS (Agent Management System): It is the agent in charge of the supervision and control over access and use of the platform. In addition, it provides the white pages' service, that is, it is responsible for the authentication of the agents and the control of records, ensuring that each agent in the platform has a unique name.

- DF (Directory Facilitator): It is the agent that provides the service of yellow pages. This is where every system agent can $\log$ the services it offers. Through the DF agent, an agent can find other agents in the system who provide the services needed to achieve their goals.

- RMA (Remote Monitoring Agent): It is the agent that allows to control the rest of agents in a platform. It provides a graphical interface that facilitates monitoring and control functions.

In addition to the agents provided by JADE, the prototype has its own agents (Fig 1):

- User Agent, it has the task of representing a user seeking to satisfy its preferences. Its mission is to observe the environment through the proposed model and compare the measured values with the target values that satisfy the user. In case the measured values vary more than a certain value (threshold) the agent has the obligation to make the requests to the coordinating agent to alter the environment seeking to favor the preferences of its user. 
- Thermal Agent, it is the agent that communicates directly with the environment and builds a representation of the environment. The role of this agent is to have knowledge of the temperature to inform other agents in the system.

- Corresponding Agent, this agent behaves as moderator or jury during negotiations between User Agents. It receives requests from user agents and calculates the comfort temperature based on the Equation (1) in order to accommodate each user.

\subsection{Validation}

This subsection describes the development of an algorithm capable of controlling the operation of air conditioning (AC) units in order to minimize use discomfort through interaction with a control platform. The operation of the algorithm is logically based on the feedback (positive or negative) from the air conditioning control decision depending on whether there is a negative/positive feedback from the user.

The current AC temperature is read and the AC status is checked (whether it has been changed by the user or remains

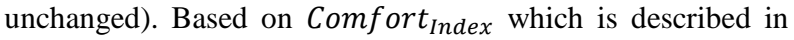
Equation (1) and used to set the comfort temperature for each user:

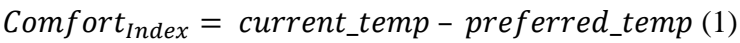

This index should be very close to the preferred temperature, which is entered by the user.

When $\operatorname{Comfort}_{\text {Index }} \approx 0$, then set:

$$
\text { Comfort }_{\text {Temp }}=\text { Preferred }_{\text {Temp }}
$$

The operating scheme of the algorithm is shown is Figure 3.

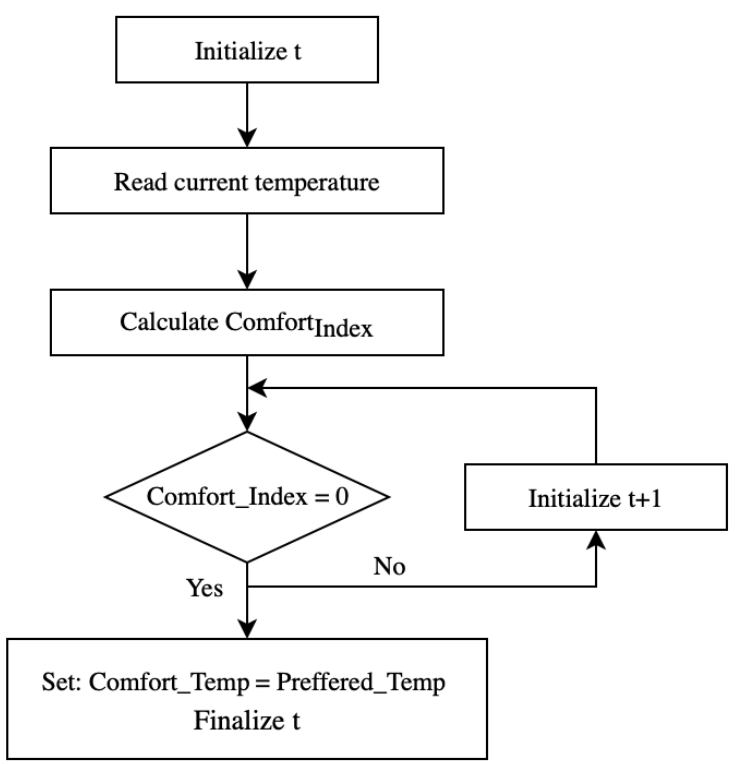

Fig 3: Operation scheme of the proposed algorithm

On the other hand, Fig 4 shows the sequence diagram provided by the JADE sniffer agent that describes the main sequence of the system, where the user agent has request to change the environment to the Coordinating Agent. Once the coordinating agent receives the request, it sends in response a proposal to change the environment to which the agents accept and proceed to execute the action requested and reports the success or failure of the action to the involved user agent.

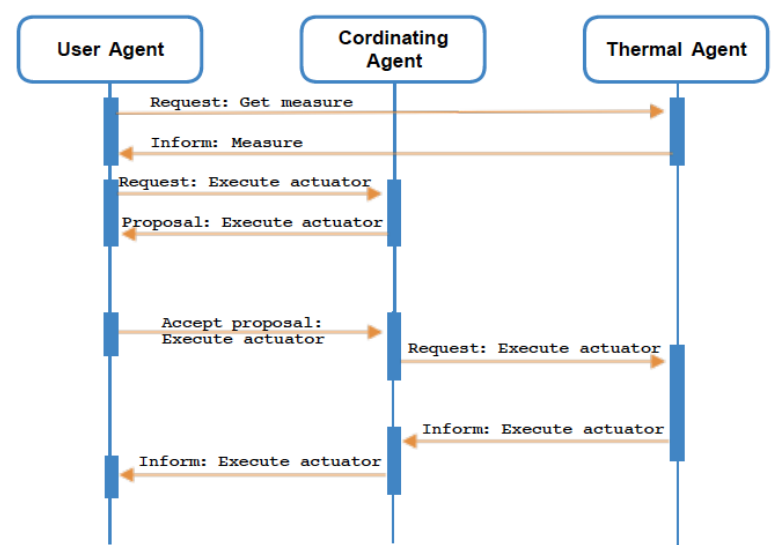

Fig 4: Sequence Diagram - Negotiation between agents

\section{CONCLUSION}

The artificial intelligence techniques have been used in many decisions, controls and automation systems over last decades. Systems for automating buildings have been no exception. In this direction, the control system architecture proposed in this paper contributes to the field of the optimization of intelligent buildings, transforming them into a dynamic space, considering standards of comfort and satisfaction for its occupants.

The performed tests allowed us to conclude that, at the very close comfort level of the occupant, the system obtained good performances by stabilizing the room temperature very close to the comfort temperature chosen by the user, taking into account the comfort level, minimum and maximum discomfort temperatures.

In this sense, the inherent skills of intelligent systems that their environment plays a very important role in achieving high system optimization. This is because the rules-based agents' system adequately captures the dependencies and interactions between components located in different sectors, thus facilitating the management of the process of automation.

\section{REFERENCES}

[1] S. Sharples, V. Callaghan, and G. Clarke, "A multi-agent architecture for intelligent building sensing and control," Sensor Review, vol. 19, no. 2, pp. 135-140, Jun. 1999.

[2] D. L. Poole, A. K. Mackworth, and R. Goebel, Computational intelligence: a logical approach, vol. 1. Oxford University Press New York, 1998.

[3] S. J. Russell and P. Norvig, Artificial intelligence: a modern approach. Malaysia; Pearson Education Limited, 2016.

[4] M. Wooldridge and N. R. Jennings, "Intelligent agents: Theory and practice," The knowledge engineering review, vol. 10, no. 2, pp. 115-152, 1995.

[5] G. H. Brundtland, "Our common future-Call for action," Environmental Conservation, vol. 14, no. 4, pp. 291-294, 1987.

[6] International Energy Agency, Transition to sustainable buildings: Strategies and opportunities to 2050. Paris, France: OECD/IEA, 2013.

[7] I. Abdennadher, N. Khabou, I. B. Rodriguez, and M. Jmaiel, "Designing energy efficient Smart Buildings in ubiquitous environments," in 2015 15th International 
Conference on Intelligent Systems Design and Applications (ISDA), 2015, pp. 122-127.

[8] B. Qolomany et al., "Leveraging Machine Learning and Big Data for Smart Buildings: A Comprehensive Survey," Apr. 2019.

[9] S. BROWN, "Graduating from smart to intelligent buildings," Consulting-Specifying Engineer, pp. 28-34, 2015.

[10] D. Kolokotsa, "The role of smart grids in the building sector," Energy and Buildings, vol. 116, pp. 703-708, 2016.

[11] H. Hagras, V. Callaghan, M. Colley, and G. Clarke, "A Hierarchical Fuzzy-Genetic Multi-Agent Architecture for Intelligent Buildings Learning, Adaptation and Control," vol. 150 , p. 17, 2003.

[12] S. D. Smitha and F. M. Chacko, "Intelligent energy management in smart and sustainable buildings with multi-agent control system," in 2013 International MutliConference on Automation, Computing, Communication, Control and Compressed Sensing (iMac4s), Kottayam, 2013, pp. 190-195.

[13] D. J. Cook, M. Youngblood, and S. K. Das, "A multiagent approach to controlling a smart environment," in Designing smart homes, Springer, 2006, pp. 165-182.

[14] A. Rogers, S. Maleki, S. Ghosh, and N. R. Jennings, "Adaptive home heating control through Gaussian process prediction and mathematical programming," 2011.

[15] D. Irwin, S. Barker, A. Mishra, P. Shenoy, A. Wu, and J. Albrecht, "Exploiting home automation protocols for load monitoring in smart buildings," in Proceedings of the Third ACM Workshop on Embedded Sensing Systems for Energy-Efficiency in Buildings, 2011, pp. 712.

[16] S. Abras, S. Pesty, S. Ploix, and M. Jacomino, "Advantages of MAS for the resolution of a power management problem in smart homes," in Advances in Practical Applications of Agents and Multiagent Systems, Springer, 2010, pp. 269-278.

[17] L. Klein et al., "Coordinating occupant behavior for building energy and comfort management using multiagent systems," Automation in Construction, vol. 22, pp. 525-536, Mar. 2012.

[18] P. H. Shaikh, N. Mohd. Nor, P. Nallagownden, and I. Elamvazuthi, "Intelligent Optimized Control System for Energy and Comfort Management in Efficient and Sustainable Buildings," Procedia Technology, vol. 11, pp. 99-106, 2013.

[19] Z. Wang, R. Yang, and L. Wang, "Multi-agent control system with intelligent optimization for smart and energy-efficient buildings," in IECON 2010 - 36th
Annual Conference on IEEE Industrial Electronics Society, Glendale, AZ, USA, 2010, pp. 1144-1149.

[20] Z. Wang, R. Yang, and L. Wang, "Intelligent multi-agent control for integrated building and micro-grid systems," in ISGT 2011, Anaheim, CA, USA, 2011, pp. 1-7.

[21] N. Minar, M. Gray, O. Roup, R. Krikorian, and P. Maes, "Hive: Distributed agents for networking things," in Proceedings. First and Third International Symposium on Agent Systems Applications, and Mobile Agents, 1999, pp. 118-129.

[22] E. M. Kan, K. Yadanar, N. H. Ling, Y. Soh, and N. Lin, "Multi-agent control system with intelligent optimization for building energy management," in Proceedings of the 18th Asia Pacific Symposium on Intelligent and Evolutionary Systems-Volume 2, 2015, pp. 505-518.

[23] D. E. Claridge, B. Abushakra, J. S. Haberl, and A. Sreshthaputra, "Electricity diversity profiles for energy simulation of office buildings," 2004

[24] C. Liao and P. Barooah, "An integrated approach to occupancy modeling and estimation in commercial buildings," in Proceedings of the 2010 American Control Conference, 2010, pp. 3130-3135.

[25] F. Haldi, "Towards a unified model of occupants' behaviour and comfort for building energy simulation," EPFL, 2010.

[26] M. Muratori, M. C. Roberts, R. Sioshansi, V. Marano, and G. Rizzoni, "A highly resolved modeling technique to simulate residential power demand," Applied Energy, vol. 107, pp. 465-473, 2013.

[27] A. Alfakara and B. Croxford, "Using agent-based modelling to simulate occupants' behaviours in response to summer overheating," in Proceedings of the Symposium on Simulation for Architecture \& Urban Design, 2014, p. 13.

[28] J. Langevin, J. Wen, and P. L. Gurian, "Simulating the human-building interaction: Development and validation of an agent-based model of office occupant behaviors," Building and Environment, vol. 88, pp. 27-45, Jun. 2015.

[29] A. Kashif, "Modélisation du comportement humain réactif et délibératif avec une approche multi-agent pour la gestion énergétique dans le bâtiment," $\mathrm{PhD}$ Thesis, Grenoble, 2014.

[30] M. Sierhuis, W. J. Clancey, and R. J. Van Hoof, "Brahms: a multi-agent modelling environment for simulating work processes and practices," International Journal of Simulation and Process Modelling, vol. 3, no. 3, pp. 134-152, 2007.

[31] F. L. Bellifemine, G. Caire, and D. Greenwood, Developing multi-agent systems with JADE, vol. 7. John Wiley \& Sons, 2007. 